\title{
Spontaneous Tilting Transition in
}

\section{Liquid-Crystalline Polymer Brushes}

\author{
S. Blaber, ${ }^{\dagger}$ N. M. Abukhdeir,,+9 and M. W. Matsen ${ }^{* \dagger, \pm, \uparrow}$ \\ $\dagger$ Department of Physics 83 Astronomy, University of Waterloo, Waterloo, Ontario, Canada \\ $\ddagger$ Department of Chemical Engineering, University of Waterloo, Waterloo, Ontario, Canada \\ \Waterloo Institute for Nanotechnology, University of Waterloo, Waterloo, Ontario, \\ Canada \\ E-mail: mwmatsen@uwaterloo.ca
}

\begin{abstract}
The equilibrium phase behavior of main-chain liquid-crystalline polymer brushes in good solvent is investigated using self-consistent field theory. The calculation assumes semi-dilute brushes, where the grafting density is sufficient to cause overlap among the polymers but low enough to allow the solvent to be treated implicitly. The polymers are modeled as semi-flexible worm-like chains with Maier-Saupe interactions. Previous calculations based on freely-jointed chains have predicted that extended brushes collapse into folded nematic brushes, as the liquid-crystalline interaction strength is increased. For the more sophisticated model of worm-like chains, which penalizes hairpin folds, we find that extended brushes become unstable with respect to tilting prior to the onset of folding. This implies that the brushes spontaneously collapse by a symmetry-breaking transition, where the chains tilt rather than fold.
\end{abstract}




\section{Introduction}

Grafting polymers to a surface provides a simple and convenient way of modifying its properties. ${ }^{1-3}$ Much of the focus has been on densely grafted brushes in good solvent, where the chains stretch out in order to maximize their contact with the solvent. Early theoretical descriptions by Alexander ${ }^{4}$ and by de Gennes ${ }^{5}$ were based on scaling theories that assumed uniform stretching with all the chain ends at the outer edge of the brush, resulting in a step concentration profile. Milner, Witten and Cates ${ }^{6}$ later used an analytical strong-stretching theory (SST) for Gaussian chains to show that the chain ends are distributed throughout the brush, resulting in a parabolic concentration profile. Numerical self-consistent field theory (SCFT) for finite degrees of stretching has since revealed a couple notable deviations from the parabolic shape, in particular a depletion layer near the grafting surface and an

exponentially-decaying tail at the outer edge of the brush. ${ }^{7,8}$ This has brought the theory into good agreement with simulations ${ }^{9,10}$ and experiments. ${ }^{11-15}$ Nevertheless, this simple model is not without its limitations.

Brush calculations typically ignore the orientational interactions between polymer chains, which generally favor parallel alignment. Even though they are relatively weak for flexible polymers, they still enhance the degree of stretching. ${ }^{16}$ More interesting, however, are the stiffer liquid-crystalline (LC) polymers that tend to align into a nematic phase. Using an Alexander-de Gennes type calculation, Halperin and Williams ${ }^{17,18}$ demonstrated how LC brushes could be used to control the alignment of a liquid-crystalline solvent, an important issue for LC display technology. This potential application, along with others such as organic electronics, switches, and sensors, has spurred numerous experimental studies. ${ }^{19-29}$

Subsequent theoretical calculations have, so far, concentrated on the simpler system of LC brushes in conventional solvents. In particular, Birshtein et al. ${ }^{30}$ examined freely-jointed chains interacting by Maier-Saupe LC interactions. ${ }^{31}$ They initially solved the statistical mechanics of the brush assuming uniform stretching (i.e., the Alexander-de Gennes approximation) with the rigid segments constrained to the bonds of a simple-cubic lattice. Provided the 
solvent quality was sufficiently good, increasing the LC interactions produced a first-order transition from a conventional brush $(\mathrm{CB})$ to a collapsed liquid-crystalline brush (LCB). Kuznetsov and Chen ${ }^{32}$ performed a similar off-lattice calculation, which likewise predicted an analogous transition for good solvents.

The Birshtein group ${ }^{33-36}$ later dropped the Alexander-de Gennes approximation and instead solved the statistical mechanics using lattice SCFT. By allowing for nonuniform stretching, they found that the brush evolved from $\mathrm{CB}$ to $\mathrm{LCB}$ via the formation of a microphase-segregated brush (MSB), with an inner high-density nematic region and an outer low-density conventional brush. Computer simulations of brushes with freely-jointed polymers also observed this phase. ${ }^{37}$ In order to create a high concentration while maintaining nematic order, the polymer chains reverse direction multiple times resulting in a series of hairpin folds. Naturally, freely-jointed chains omit the usual penalty for hairpin folds. ${ }^{17,18,38}$ Including this penalty may very well cause the brush to increase its concentration in a different way.

Indeed, simulations of short-chain LC molecules with a bending penalty have observed tilting. ${ }^{39-42}$ Although some of the calculations by Birshtein and coworkers ${ }^{33}$ did in fact restrict the bending between consecutive chain segments, their lattice nevertheless precluded the possibility of tilting. Off-lattice SCFT calculations have been performed by Deng et al., ${ }^{43}$ for worm-like chains ${ }^{44,45}$ with Onsager LC interactions. ${ }^{46}$ However, that study only considered azimuthally-symmetric phases (i.e., invariant with respect to rotations about axes normal to the grafting surface), which likewise precluded tilted phases.

A few studies have allowed for the possibility of tilting when considering LC polymeric brushes subjected to external forces, either shear ${ }^{47}$ or compression. ${ }^{48-50}$ However, it seems that the possibility of spontaneous tilting due to strong LC interactions has so far been overlooked. Therefore, we reconsider the SCFT for worm-like chains by Deng et al. ${ }^{43}$ allowing for breaking of the azimuthal symmetry. We also switch to the more general Maier-Saupe interactions, rather than the Onsager interactions specific to hard-core cylindrical segments. 


\section{Theory}

Here we consider a brush with $n_{p}$ polymers grafted to a flat substrate of area, $\mathcal{A}$, at $z=0$, immersed in a solvent of $n_{s}$ molecules. Each polymer has $N$ segments of length $b$, giving a total contour length of $\ell_{c}=N b$. The configuration of the $\alpha^{\prime}$ th polymer chain is specified by the space curve $\mathbf{r}_{p, \alpha}(s)$, where the backbone parameter runs from $s=0$ at the grafted end to $s=1$ at the free end. From this, we obtain the unit vector $\mathbf{u}_{p, \alpha}(s)=\ell_{c}^{-1} \frac{d}{d s} \mathbf{r}_{p, \alpha}(s)$ specifying the orientation of the chain. It then follows that the dimensionless probability density of finding a segment at position $\mathbf{r}$ with orientation $\mathbf{u}$ is

$$
\hat{\phi}_{p}(\mathbf{r}, \mathbf{u})=\frac{N}{\rho_{0}} \sum_{\alpha=1}^{n_{p}} \int_{0}^{1} d s \delta\left(\mathbf{r}-\mathbf{r}_{p, \alpha}(s)\right) \delta\left(\mathbf{u}-\mathbf{u}_{p, \alpha}(s)\right)
$$

where $\rho_{0}^{-1}$ is the volume of an individual polymer segment. Integrating the distribution over all orientations provides the polymer concentration,

$$
\hat{\phi}_{p}(\mathbf{r})=\int d \mathbf{u} \hat{\phi}_{p}(\mathbf{r}, \mathbf{u})
$$

where $d \mathbf{u}=\sin (\theta) d \theta d \varphi$. Likewise, the dimensionless solvent concentration is

$$
\hat{\phi}_{s}(\mathbf{r})=v_{s} \sum_{\alpha=1}^{n_{s}} \delta\left(\mathbf{r}-\mathbf{r}_{s, \alpha}\right)
$$

where $v_{s}$ is the volume of each solvent molecule and $\mathbf{r}_{s, \alpha}$ is the position of the $\alpha^{\prime}$ th solvent

molecule. Assuming incompressibility, the solvent concentration is given by $\hat{\phi}_{s}(\mathbf{r})=1-\hat{\phi}_{p}(\mathbf{r})$, which allows the interaction energy, $U_{\text {int }}$, to be expressed solely in terms of the polymer concentration. Here, we choose the Maier-Saupe interaction energy

$$
\frac{U_{\mathrm{int}}}{k_{B} T}=\frac{\rho_{0}}{2} \int d \mathbf{r} d \mathbf{u} d \mathbf{u}^{\prime} \hat{\phi}_{p}(\mathbf{r}, \mathbf{u}) f\left(\mathbf{u}, \mathbf{u}^{\prime}\right) \hat{\phi}_{p}\left(\mathbf{r}, \mathbf{u}^{\prime}\right)
$$


where

$$
f\left(\mathbf{u}, \mathbf{u}^{\prime}\right)=\nu_{0}-\nu_{2} P_{2}\left(\mathbf{u} \cdot \mathbf{u}^{\prime}\right)
$$

involves the Legendre polynomial $P_{2}(x)=\left(3 x^{2}-1\right) / 2$.

The model is solved using SCFT with the polymers treated as worm-like chains. ${ }^{44,45}$ As such, the energy of an individual polymer takes the form

$$
\frac{H_{p}}{k_{\mathrm{B}} T}=\int_{0}^{1} d s\left[\frac{\kappa}{2 N}\left|\frac{d \mathbf{u}_{p, \alpha}}{d s}\right|^{2}+w\left(\mathbf{r}_{p, \alpha}, \mathbf{u}_{p, \alpha}\right)\right]
$$

The first term is the energy penalty due to bending of the polymer chain. The dimensionless modulus, $\kappa$, controls the persistence length, $\ell_{p}=b \kappa$, and statistical segment length, $a=$ $b \sqrt{2 \kappa}$. The second term is the energy from the self-consistent field

$$
w(\mathbf{r}, \mathbf{u})=N \int d \mathbf{u}^{\prime} f\left(\mathbf{u}, \mathbf{u}^{\prime}\right) \phi_{p}\left(\mathbf{r}, \mathbf{u}^{\prime}\right)
$$

which represents interactions with the ensemble-average distribution, $\phi_{p}\left(\mathbf{r}, \mathbf{u}^{\prime}\right) \equiv\left\langle\hat{\phi}_{p}\left(\mathbf{r}, \mathbf{u}^{\prime}\right)\right\rangle$.

Within the mean-field approximation, the entropy of the solvent molecules can be expanded as

$$
\begin{aligned}
-\frac{S_{s}}{k_{B}} & =\frac{1}{v_{s}} \int d \mathbf{r} \phi_{s} \ln \phi_{s} \\
& =\frac{1}{v_{s}} \int d \mathbf{r}\left[-\phi_{p}+\frac{\phi_{p}^{2}}{2}+\frac{\phi_{p}^{3}}{6}+\cdots\right]
\end{aligned}
$$

where $\phi_{s}(\mathbf{r}) \equiv\left\langle\hat{\phi}_{s}(\mathbf{r})\right\rangle$ and $\phi_{p}(\mathbf{r}) \equiv\left\langle\hat{\phi}_{p}(\mathbf{r})\right\rangle$ are ensemble-average concentrations. The linear term of the expansion has no effect because it integrates to a constant, and the quadratic term can be incorporated into $\nu_{0}$ for the isotropic interactions. We then assume a semi-dilute brush, where the concentration is sufficient for mean-field theory to be accurate but small enough for the higher-order terms to be ignored. As such, the effect of the solvent size, $v_{s}$, is absorbed into $\nu_{0}$, and the solvent degrees of freedom are removed from the problem. 
Furthermore, the semi-dilute approximation with implicit solvent allows the grafting density, $\sigma=n_{p} / \mathcal{A}$, to be absorbed into the scaled concentration

$$
\phi(z, \mathbf{u}) \equiv \frac{b \rho_{0}}{\sigma} \phi_{p}(\mathbf{r}, \mathbf{u})
$$

which is normalized such that

$$
\frac{1}{\ell_{c}} \int d z \phi(z)=1
$$

where $\phi(z)=\int d \mathbf{u} \phi(z, \mathbf{u})$. Note that the grafting density is assumed to be sufficient such that there is no need to constrain the lateral positions of the grafting points, which leads to ensemble-average concentrations that are independent of $x$ and $y$. In terms of the scaled concentrations, the field equation becomes

$$
w(z, \mathbf{u})=\int d \mathbf{u}^{\prime} G\left(\mathbf{u}, \mathbf{u}^{\prime}\right) \phi\left(z, \mathbf{u}^{\prime}\right)
$$

where

$$
G\left(\mathbf{u}, \mathbf{u}^{\prime}\right)=\Lambda_{0}-\Lambda_{2} P_{2}\left(\mathbf{u} \cdot \mathbf{u}^{\prime}\right)
$$

involves the reduced interaction parameters

$$
\Lambda_{0} \equiv \frac{\nu_{0} \sigma N}{b \rho_{0}} \quad \text { and } \quad \Lambda_{2} \equiv \frac{\nu_{2} \sigma N}{b \rho_{0}}
$$

The SCFT requires the statistical mechanics of a single polymer with Hamiltonian $H_{p}$, where the $s$ 'th segment is constrained at position $\mathbf{r}$ and orientation $\mathbf{u}$. The partition function of the constrained polymer can be written as a product, $q(z, \mathbf{u}, s) q^{\dagger}(z, \mathbf{u}, s)$, of partial partition functions for the two portions of chain to either side of the constraint. The $q(z, \mathbf{u}, s)$ for the portion attached to the substrate satisfies the diffusion equation ${ }^{45,51,52}$

$$
\frac{\partial q}{\partial s}+\ell_{c} u_{z} \frac{\partial q}{\partial z}=\frac{\ell_{c}}{2 \ell_{p}} \nabla_{\mathbf{u}}^{2} q-w q
$$


with an initial condition $q(z, \mathbf{u}, 0)=g(z, \mathbf{u})$ related to the grafting potential. The $q^{\dagger}(z, \mathbf{u})$ for the portion with the free end satisfies Eq. (14) with the left-hand side multiplied by -1 subject to $q^{\dagger}(z, \mathbf{u}, 1)=1$. The impenetrability of the substrate is enforced by applying Dirichlet boundary conditions, $q(0, \mathbf{u}, s)=q^{\dagger}(0, \mathbf{u}, s)=0$.

Once the partial partition functions are known, the distribution of polymer is given by

$$
\phi(z, \mathbf{u})=\frac{\ell_{c}}{\mathcal{Z}} \int_{0}^{1} d s q(z, \mathbf{u}, s) q^{\dagger}(z, \mathbf{u}, s)
$$

where

$$
\mathcal{Z}=\int d z d \mathbf{u} q(z, \mathbf{u}, s) q^{\dagger}(z, \mathbf{u}, s)
$$

is the partition function of an unconstrained chain. Note that the integration in Eq. (16) is independent of $s$. The field must be adjusted such that $\phi(z, \mathbf{u})$ satisfies Eq. (11).

The orientational order of the polymer chains can be characterized by the $\mathcal{Q}$-tensor, ${ }^{51,53}$

$$
\mathcal{Q}_{i, j}(z)=\frac{3}{2 \phi(z)} \int d \mathbf{u}\left(u_{i} u_{j}-\frac{\delta_{i, j}}{3}\right) \phi(z, \mathbf{u})
$$

where $i, j \in\{x, y, z\}$. The Kronecker delta is included to make the tensor traceless (i.e., $\operatorname{Tr} \mathcal{Q}=0)$, such that it reduces to the diagonal form

$$
\mathcal{Q}_{i, j}=\frac{3 \mathcal{S}}{2}\left(n_{i} n_{j}-\frac{\delta_{i, j}}{3}\right)+\frac{\mathcal{P}}{2}\left(l_{i} l_{j}-m_{i} m_{j}\right)
$$

where $\mathbf{n}, \mathbf{l}$ and $\mathbf{m}$ are orthonormal eigenvectors. The largest eigenvalue, $\mathcal{S}(z)$, and its corresponding eigenvector, $\mathbf{n}(z)$, are referred to as the uniaxial order parameter and the nematic director, respectively. $\mathcal{S}(z)$ varies from zero for an isotropic phase to one for a fully-ordered nematic phase. The difference between the two smallest eigenvalues defines the biaxial order parameter, $\mathcal{P}(z)$.

The field Eq. (11) generally has multiple solutions corresponding to different metastable phases, and so we need to evaluate their free energies in order to determine which is stable. 
In mean-field theory, the free energy is given by

$$
F=-n_{p} k_{B} T \ln \mathcal{Z}-\frac{1}{\ell_{c}} \int d z U(z)
$$

The logarithmic term provides the free energy of $n_{p}$ chains in the mean field, which double counts the interaction energy. To correct for this, the second term subtracts $U_{\text {int }}$, where the energy density can be expressed as

$$
\begin{aligned}
\frac{U(z)}{n_{p} k_{B} T} & =\frac{1}{2} \int d \mathbf{u} d \mathbf{u}^{\prime} \phi(z, \mathbf{u}) G\left(\mathbf{u}, \mathbf{u}^{\prime}\right) \phi\left(z, \mathbf{u}^{\prime}\right) \\
& =\left(\frac{\Lambda_{0}}{2}-\frac{\Lambda_{2}}{3} \operatorname{Tr} \mathcal{Q}^{2}(z)\right) \phi^{2}(z) \\
& =\frac{1}{2}\left[\Lambda_{0}-\Lambda_{2}\left(\mathcal{S}^{2}(z)+\frac{\mathcal{P}^{2}(z)}{3}\right)\right] \phi^{2}(z)
\end{aligned}
$$

As usual, the SCFT is solved numerically. For the diffusion Eq. (14), we use a generalization of the algorithm in Ref. 54 that involves finite differences, $\Delta z=\ell_{c} / 800$ and $\Delta s=1 / 800$, for the spatial and the contour variables, respectively, and an expansion in spherical harmonics, $Y_{\ell, m}(\theta, \varphi)$ with $\ell \leq 10$, for the orientation. To avoid numerical difficulties, the grafting function is chosen as $g(z, \mathbf{u})=\exp \left(-z^{2} / \xi^{2}\right)$, corresponding to a freely-jointed graft with a small but finite range of $\xi=\ell_{c} / 32$. The field Eq. (11) is solved iteratively using Anderson mixing, ${ }^{55}$ which has proven to be very effective for other polymeric systems. ${ }^{56,57}$ Naturally, the expansion in spherical harmonics allows integrals over $\mathbf{u}$ to be performed analytically; integrals over $z$ and $s$ are evaluated using the simple trapezoid rule.

\section{Results}

We begin by examining azimuthally-symmetric solutions of the field Eq. (11), $w_{0}\left(z, u_{z}\right)$ where $u_{z}=\cos (\theta)$. This is achieved by restricting the expansion in spherical harmonics, $Y_{\ell, m}(\theta, \varphi)$, to $m=0$. Figure 1 shows the concentration profile, $\phi(z)$, as the LC interaction strength, $\Lambda_{2}$, is increased for worm-like chains of different flexibility, $\ell_{c} / \ell_{p}$. In the absence of 

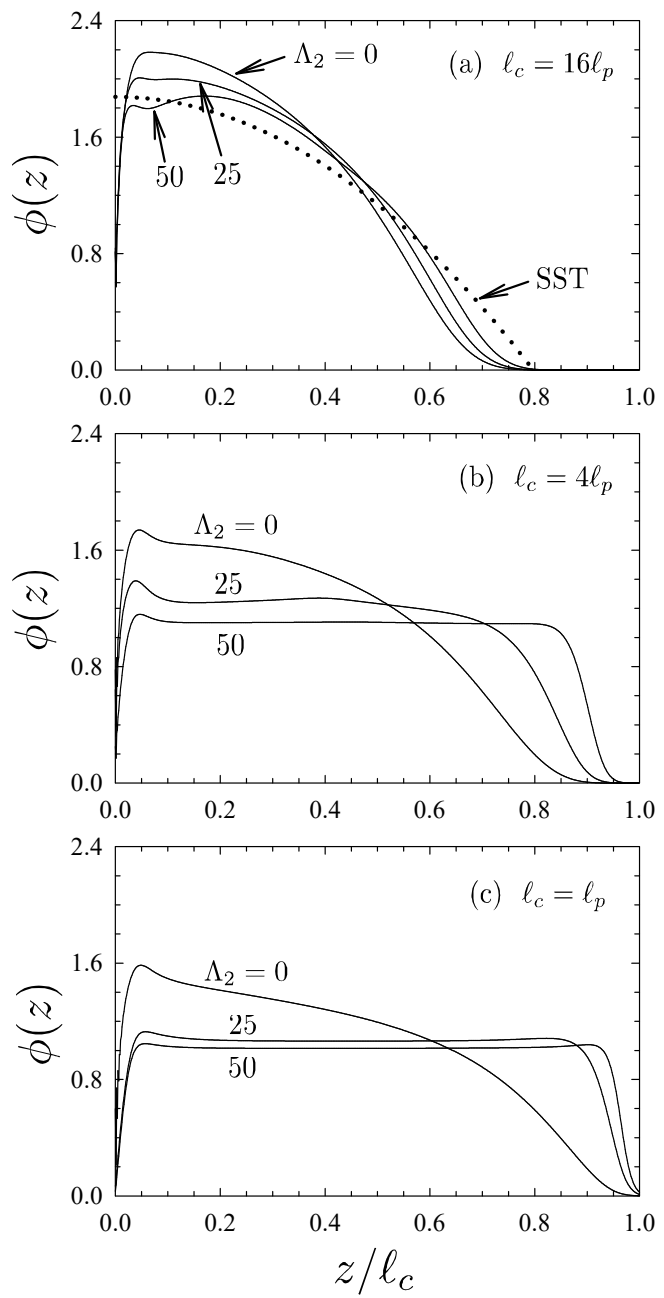

Figure 1: Concentration profiles, $\phi(z)$, corresponding to $\Lambda_{0}=10$ and $\Lambda_{2}=0,25$, and 50, calculated for worm-like chains of (a) $\ell_{c}=16 \ell_{p}$, (b) $\ell_{c}=4 \ell_{p}$, and (c) $\ell_{c}=\ell_{p}$. The dotted curve in (a) denotes the parabolic profile predicted by SST for Gaussian chains with no LC interactions. 
LC interactions (i.e., $\Lambda_{2}=0$ ), the most flexible polymers in Fig. 1(a) have a parabolic-like profile, similar to that predicted by SST for Gaussian chains. However, there is a considerable quantitative deviation from the SST prediction (dotted curve), which can be attributed to the fact that the SST brush height, ${ }^{6,8}$

$$
L=\left(\frac{8 \Lambda_{0} \ell_{p}}{\pi^{2} \ell_{c}}\right)^{1 / 3} \ell_{c}
$$

is nearly $80 \%$ of the polymer contour length, $\ell_{c}$. Nevertheless, consistent with previous results in Ref. 43, good quantitative agreement does occur for more flexible polymers, provided $\ell_{c}$ is at least twice $L$ which in turn is at least double $R_{0} \equiv a N^{1 / 2}=\left(2 \ell_{p} \ell_{c}\right)^{1 / 2}$. In any case, increasing the LC interactions and reducing the flexibility both cause the profile to deviate further from the parabolic profile toward a step-like profile of height $\ell_{c}$. For our most rigid polymer with the strongest LC interactions in Fig. 1(c), the concentration profile corresponds to more or less fully extended chains. In fact, the concentration extends slightly beyond the total contour length of the polymer, which is only possible due to the finite width, $\xi$, of our grafting function, $g(z, \mathbf{u})$.

To assess the orientation of the polymer chains, we diagonalize the tensor, $\mathcal{Q}_{i, j}(z)$, defined in Eq. (17). Under azimuthal symmetry, the tensor has a single eigenvalue, $\lambda_{\perp}(z)$, corresponding to an eigenvector in the $z$ direction, and two degenerate eigenvalues, $\lambda_{\|}(z)$, corresponding to eigenvectors in the $x-y$ plane. Given that $\mathcal{Q}_{i, j}(z)$ is traceless, it follows that $\lambda_{\perp}(z)+2 \lambda_{\|}(z)=0$, and therefore $\lambda_{\perp}(z)$ and $\lambda_{\|}(z)$ have opposite signs. In general, $\lambda_{\perp}(z)$ is the positive eigenvalue, which implies that the nematic director, $\mathbf{n}(z)$, is normal to the grafting surface with a uniaxial order parameter of $\mathcal{S}(z)=\lambda_{\perp}(z)$ and a biaxial order parameter of $\mathcal{P}(z)=0$. However, as illustrated in Fig. 2, there is a narrow surface region, $0<z<z^{*}$, where $\mathbf{n}(z)$ lies in the $x$-y plane, $\mathcal{S}(z)=\lambda_{\|}(z)$, and $\mathcal{P}(z)=3 \lambda_{\|}(z)$. This may seem counterintuitive given that the grafted chains extend outward through this region. However, they do not contribute much to the surface concentration because of their 
perpendicular orientation. On the other hand, chains that, for example, loop back to the surface tend to have a large fraction of their contour parallel to the surface, and thus contribute considerably to the surface concentration despite their relatively small population. Note though that the quantitative details of the surface layer are significantly affected by the choice of grafting function, $g\left(z, u_{z}\right)$, and so we do not focus on it in this study.

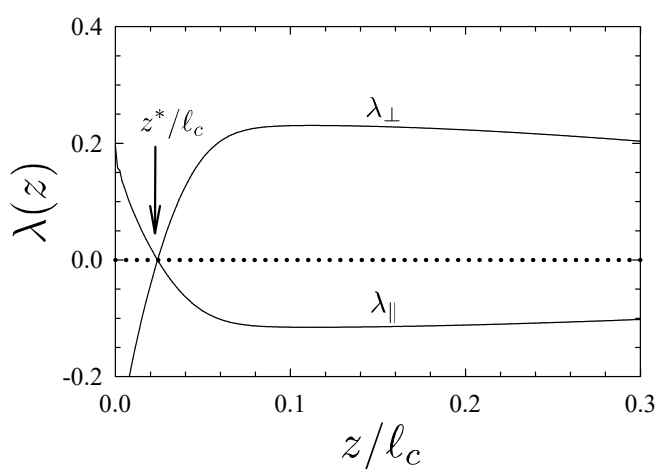

Figure 2: Eigenvalues of the $\mathcal{Q}$-tensor for worm-like chains of $\ell_{c}=16 \ell_{p}$ and interaction parameters of $\Lambda_{0}=10$ and $\Lambda_{2}=25$. The eigenvalue $\lambda_{\perp}(z)$ corresponds to an eigenvector in the $z$ direction and the degenerate eigenvalue $\lambda_{\|}(z)$ corresponds to eigenvectors in the $x-y$ plane. Their crossing point defines $z^{*}$.

Figure 3 shows the dependence of $\mathcal{S}(z)$ on the system parameters. Note that it becomes computationally difficult to determine $\mathcal{S}(z)$ as the polymer concentration approaches zero, and so the curves are only plotted for $\phi(z) \gtrsim 0.01$. As expected, $\mathcal{S}(z)$ is smallest for our most flexible chains, $\ell_{c}=16 \ell_{p}$, without LC interactions, $\Lambda_{2}=0$. Reducing the flexibility, $\ell_{c} / \ell_{p}$, and increasing the LC interactions, $\Lambda_{2}$, both cause an increase in orientational order. Indeed, $\mathcal{S}(z)$ approaches the maximum allowed value of one for our most rigid polymer, $\ell_{c}=\ell_{p}$, with the strongest LC interactions, $\Lambda_{2}=50$. In all cases, the surface region (i.e., $z<z^{*}$ ) narrows as the LC interaction strength increases, which accounts for the widening of the depletion in $\phi(z)$ next to the surface (see Fig. 1).

The isotropic and LC interactions enter $U(z)$ in Eq. (20) with opposite signs, and thus they compete against each other. Positive $\Lambda_{0}$ favors low $\phi(z)$ (i.e., extended brushes), whereas positive $\Lambda_{2}$ favors high $\phi(z)$ (i.e., collapsed brushes). One way of increasing the polymer concentration is for the polymer chains to fold. Indeed, a self-consistent field solution for 

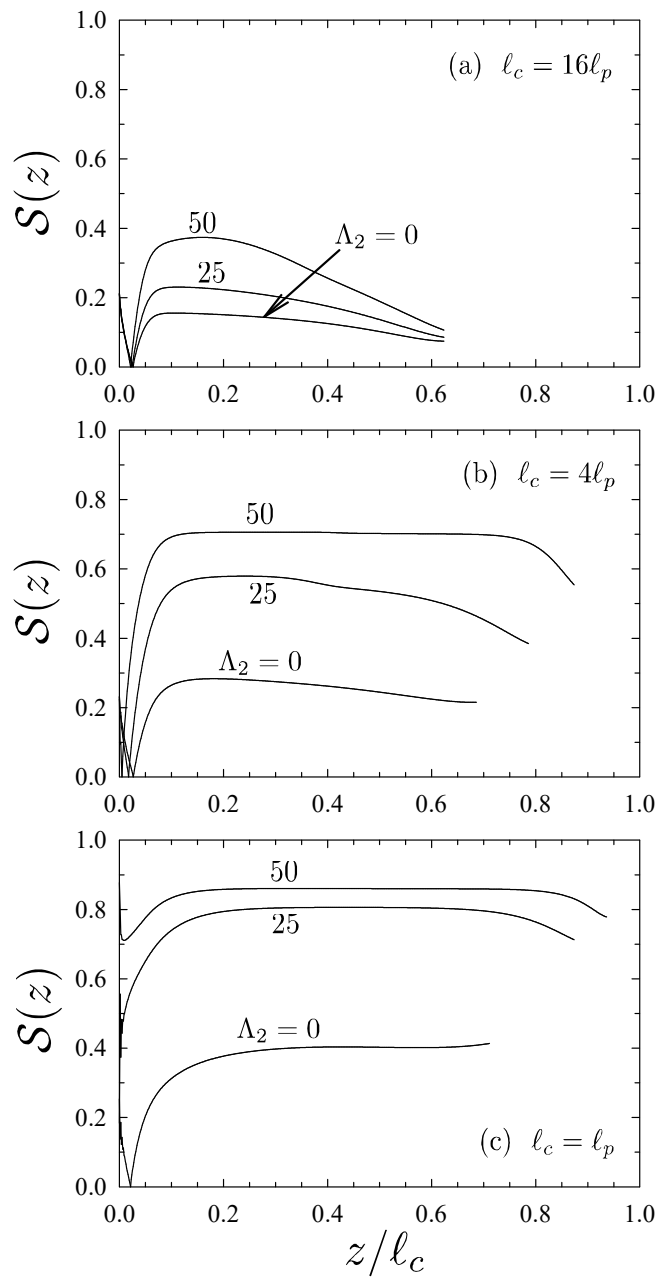

Figure 3: Uniaxial order parameter, $\mathcal{S}(z)$, corresponding to $\Lambda_{0}=10$ and $\Lambda_{2}=0$, 25, and 50, calculated for worm-like chains of (a) $\ell_{c}=16 \ell_{p}$, (b) $\ell_{c}=4 \ell_{p}$, and (c) $\ell_{c}=\ell_{p}$. 
folded polymers emerges once $\Lambda_{2}$ is sufficiently large. Figure 4(a) compares its polymer concentration to that of the extended state. Naturally, folding reduces the height of the brush by half and doubles the concentration. To confirm the configuration of the polymer chains, Figs. 4(b) and 4(c) examines the concentration distribution,

$$
\phi(z, s)=\frac{\ell_{c}}{\mathcal{Z}} \int d \mathbf{u} q(z, \mathbf{u}, s) q^{\dagger}(z, \mathbf{u}, s)
$$

of the different segments, $s$. The density plot for the extended state shows the segments monotonically shifting away from the grafting surface with increasing $s$, whereas the density plot for the folded state reveals a sharp hairpin fold at $s=0.5$. The reversal in direction is responsible for an elevated concentration at the outer edge of the folded brush, which is clearly evident in Fig. 4(a). Consistent with the high concentration of folds, the director, $\mathbf{n}(z)$, switches from perpendicular to parallel beyond the point denoted by a solid dot.

Figure 5 demonstrates the energetic advantage of the folded state by comparing its interaction energy density, $U(z)$, to that of the extended state. In the absence of LC interactions, $U(z)$ is positive for all $z$, which favors the low concentration of the extended state. However, as $\Lambda_{2}$ increases, $U(z)$ eventually becomes negative. Given that the energy density is proportional to $\phi^{2}(z)$, folding roughly doubles this energetic advantage. Nevertheless, the transition cannot happen until $U(z)$ is sufficiently negative to compensate for the energy cost of folding. Indeed, the first-order transition to a folded state, shown in Fig. 6, occurs at $\Lambda_{2}=43.3$, which is well beyond the $\Lambda_{2} \approx 30$ where $U(z)$ first becomes negative. Naturally, as $\Lambda_{2}$ increases further, $U(z)$ is able to compensate for additional folds per chain, thus producing a series of discontinuous folding transitions. However, folding is not the most efficient way of increasing $\phi(z)$.

The least costly way of increasing $\phi(z)$ is to simply tilt the nematic director, $\mathbf{n}(z)$, since this avoids hairpin folds. However, as in the SCFT of Deng et al. ${ }^{43}$ this is prevented by our restriction to symmetric fields, $w_{0}\left(z, u_{z}\right)$. To locate the spinodal where the extended phase 

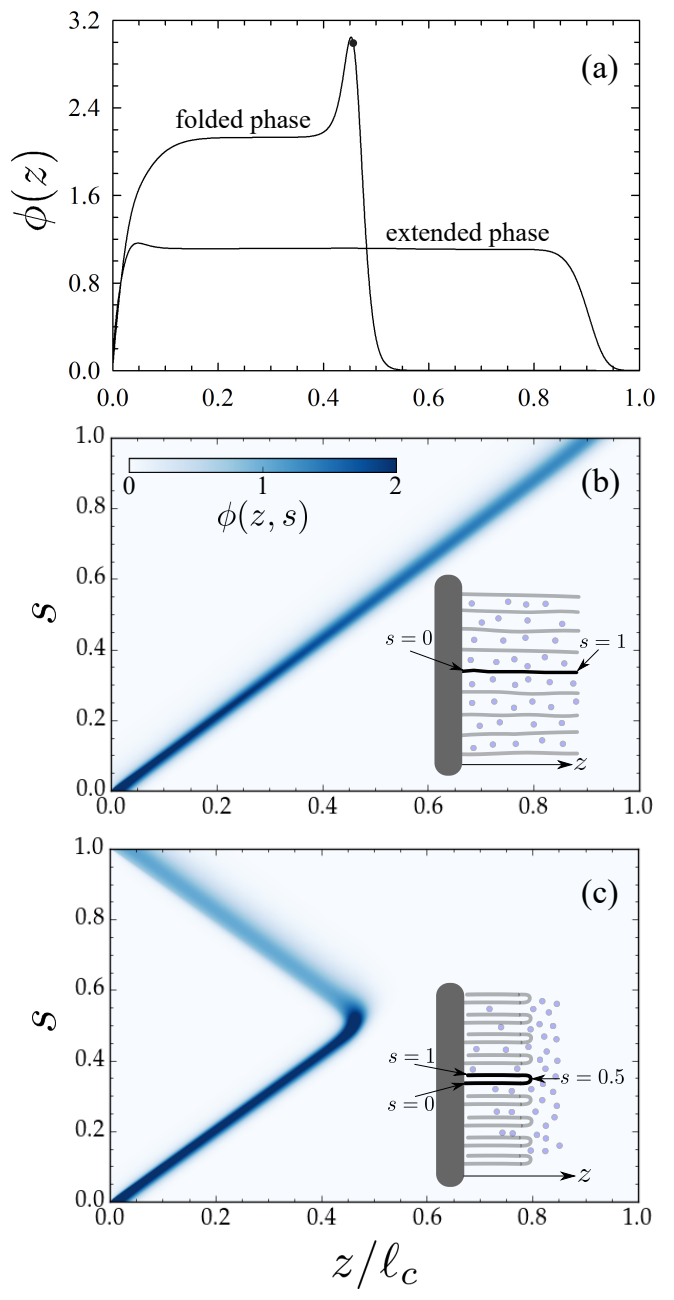

Figure 4: (a) Concentration profiles, $\phi(z)$, of the extended and folded phases, calculated for $\ell_{c}=4 \ell_{p}, \Lambda_{0}=10$, and $\Lambda_{2}=50$. The dot for the folded phase marks a change in the nematic director, $\mathbf{n}(z)$, from perpendicular to parallel. The density plots show the $s$-segment distributions, $\phi(z, s)$, in the (b) extended and (c) folded phases.

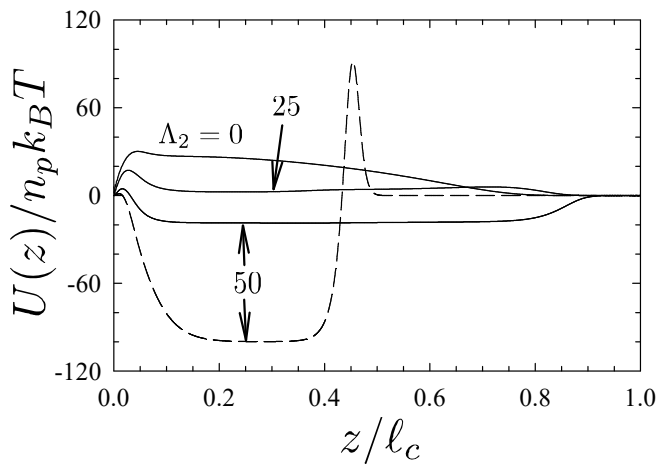

Figure 5: Energy density, $U(z)$, for an extended brush of worm-like chains with $\ell_{c}=4 \ell_{p}$ and interaction strengths of $\Lambda_{0}=10$ and $\Lambda_{2}=0,25$, and 50 (solid curves). The $U(z)$ for a folded brush is plotted but only for $\Lambda_{2}=50$ (dashed curve). 


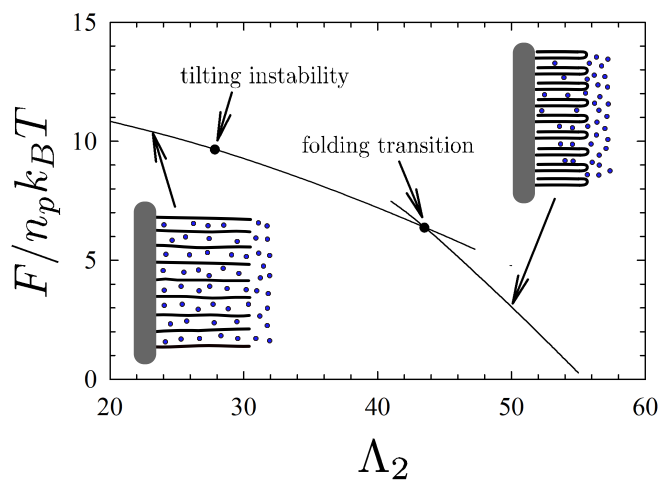

Figure 6: Free energy, $F$, as a function of $\Lambda_{2}$ for the extended and folded phases, corresponding to $\Lambda_{0}=10$ and $\ell_{c}=4 \ell_{p}$. Dots denote the first-order folding transition and the spinodal, where the extended phase becomes unstable with respect to tilting.

becomes unstable with respect to tilting, we examine the Jacobian,

$$
J_{I, I^{\prime}}=\delta_{I, I^{\prime}}-\int d \mathbf{u}^{\prime \prime} G\left(\mathbf{u}, \mathbf{u}^{\prime \prime}\right) \frac{\mathcal{D} \phi\left(z, \mathbf{u}^{\prime \prime}\right)}{\mathcal{D} w\left(z^{\prime}, \mathbf{u}^{\prime}\right)}
$$

of the field Eq. (11) with its full $\mathbf{u}$ dependence. Here, the index $I \equiv\{z, \mathbf{u}\}$ is shorthand notation for the arguments of $w(z, \mathbf{u})$ and $\phi(z, \mathbf{u})$. The functional derivative inside the integral represents the change in the polymer distribution at $z$ and $\mathbf{u}^{\prime \prime}$ due to a small change in the field at $z^{\prime}$ and $\mathbf{u}^{\prime}$, which we calculate numerically. In practise, however, we expand our functions in terms of spherical harmonics, $Y_{\ell, m}(\theta, \varphi)$, and so the indices of the Jacobian actually represent $I \equiv\{z, \ell, m\}$. Since we are interested in knowing when the azimuthal symmetry is broken, we evaluate the Jacobian for the symmetric field solution, $w_{0}\left(z, u_{z}\right)$. The solution becomes unstable when the smallest eigenvalue of $J_{I, I^{\prime}}$ approaches zero. As expected, the spinodal point where this happens, denoted by a dot in Fig. 6, preempts the folding transition.

In order to confirm that the instability corresponds to tilting, we calculate the polymer distribution, $\phi(z, \mathbf{u})$, for the field,

$$
w(z, \mathbf{u})=w_{0}\left(z, u_{z}\right)+c \delta w(z, \mathbf{u})
$$


where $\delta w(z, \mathbf{u})$ is an eigenvector corresponding to the zero eigenvalue. Note that the eigenvalue is degenerate, due to the fact that the chains can tilt in any direction. We select the eigenvector corresponding to a tilt in the $\varphi=0$ direction, normalized such that $\ell_{c}^{-1} \int d z d \mathbf{u} \delta w^{2}(z, \mathbf{u})=1$. The fact that the eigenvalue is zero implies that the field in Eq. (24) remains self-consistent for small $c$ [i.e., satisfies Eq. (11)]. Figure 7 plots the angle of the nematic director with respect to the $z$ axis, $\theta_{\mathbf{n}}(z)$, obtained from diagonalizing the $\mathcal{Q}$-tensor for different levels of asymmetry. As discussed previously in regards to Fig. 2, when the field is symmetric (i.e, $c=0$ ), the orientation switches discontinuously from $\theta_{\mathbf{n}}(z)=90^{\circ}$ to $0^{\circ}$ at $z=z^{*}$. However, once the symmetry is broken (i.e., $c>0$ ), the angle changes continuously. Furthermore, adding a larger amount of the eigenvector, $\delta w(z, \mathbf{u})$, to the symmetric solution, $w_{0}\left(z, u_{z}\right)$, increases the degree of tilt.

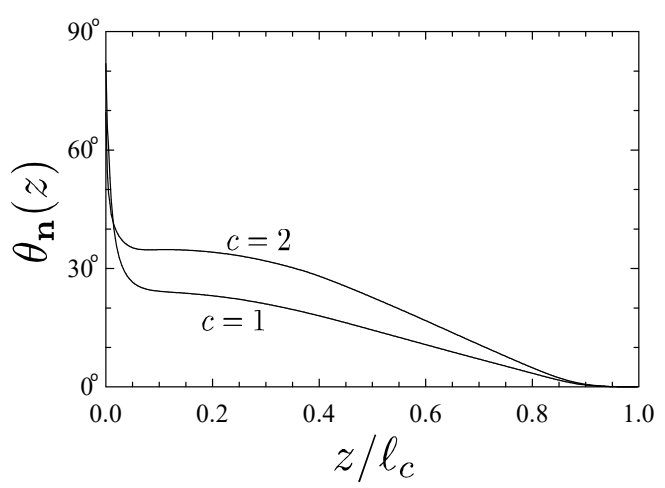

Figure 7: Tilt of the nematic director, $\mathbf{n}(z)$, as a function of distance from the grafting surface, calculated for fields, $w_{0}\left(z, u_{z}\right)+c \delta w(z, \mathbf{u})$, with different levels of broken symmetry.

The diagonalization of $\mathcal{Q}_{i, j}(z)$ also provides the uniaxial, $\mathcal{S}(z)$, and biaxial, $\mathcal{P}(z)$, order parameters plotted in Fig. 8. Naturally, the polymer concentration, $\phi(z)$, increases as the chains tilt, which in turn enhances the level of LC order as evident by the increase in $\mathcal{S}(z)$. Furthermore, the tilt of the grafting surface with respect to the nematic director, $\mathbf{n}(z)$, creates a small degree of biaxiality, as illustrated in Fig. 8(b). The increase of both $\mathcal{S}(z)$ and $\mathcal{P}(z)$ reduces the interaction energy density, $U(z)$, shown in Fig. $8(\mathrm{c})$. It is worth pointing out that $U(z)$ tends to be less negative at large $z$, which explains why the tilt angle, $\theta_{\mathbf{n}}(z)$, diminishes towards the outer edge of the brush in Fig. 7. In any case, the reduction 

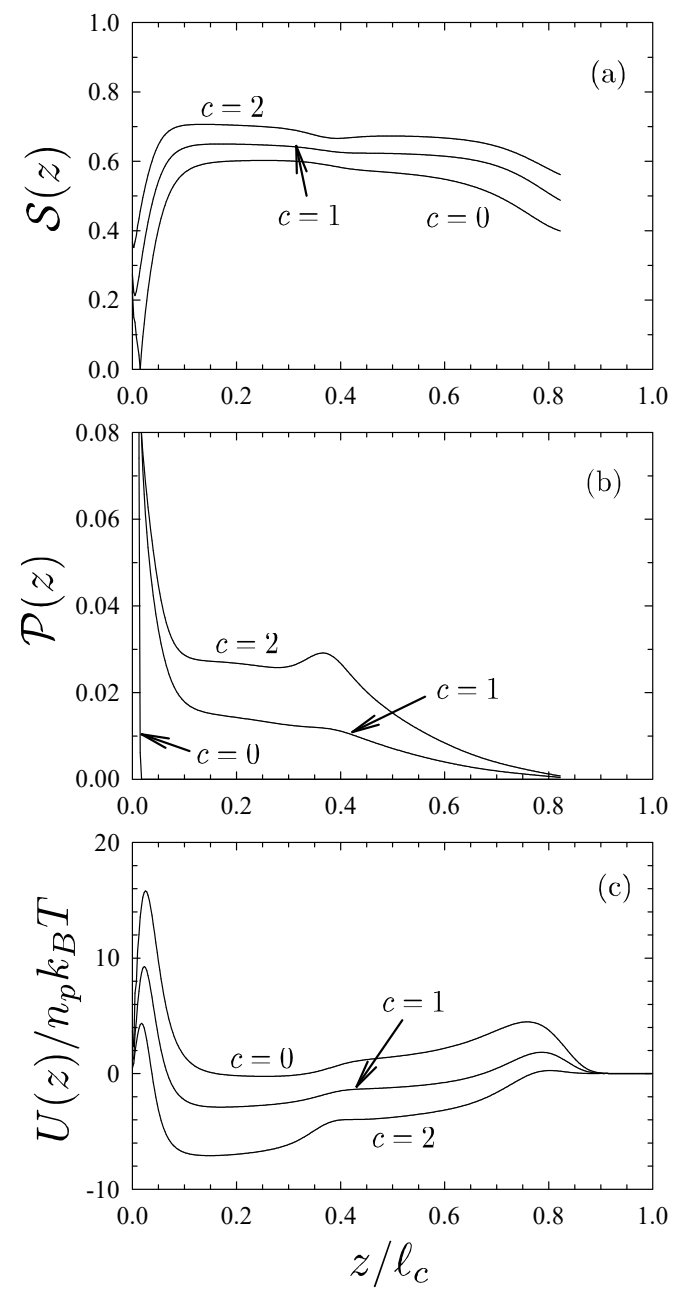

Figure 8: (a) Uniaxial order parameter, $\mathcal{S}(z)$, (b) biaxial order parameter, $\mathcal{P}(z)$, and (c) energy density, $U(z)$, corresponding to self-consistent fields, Eq. (24), with different levels of broken symmetry, calculated for $\ell_{c}=4 \ell_{p}, \Lambda_{0}=10$, and $\Lambda_{2}=27.8$. 
in the total interaction energy, $U_{\text {int }}$, causes the free energy to decrease monotonically as $c$ increases. In the absence of any mechanism countering the decrease in free energy, the tilt angle will simply jump discontinuously towards $90^{\circ}$ as soon as the spinodal is crossed. As we will discuss later, this unphysical behavior is a limitation of the semi-dilute approximation.

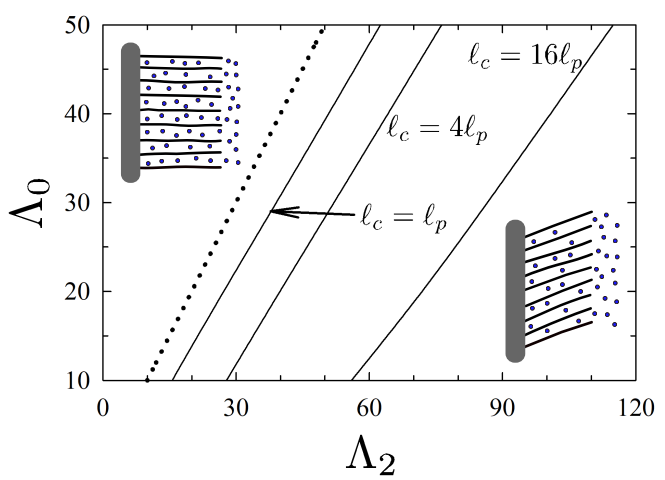

Figure 9: Diagram showing the spinodal where the extended brush becomes unstable with respect to tilting, calculated for polymers of different flexibility. The dotted line, $\Lambda_{2}=\Lambda_{0}$, denotes the minimum LC interaction required before tilting can possibly occur.

Despite the fact that the current model is insufficient to determine the equilibrium properties of the tilted phase, it does provide the boundary between it and the extended phase. Figure 9 plots the boundary in the $\Lambda_{0}-\Lambda_{2}$ plane for worm-like chains of different flexibility. The position of the spinodal roughly coincides with $\int d z U(z)=0$, where $U(z)$ is given by Eq. (20). Thus, the shift in the boundary with increasing chain stiffness (i.e., increasing $\ell_{p}\left(\ell_{c}\right)$ is readily explained by the increase in $\mathcal{S}(z)$ plotted in Fig. 3. Given that the energy density satisfies the inequality $U(z) \geq \frac{1}{2}\left(\Lambda_{0}-\Lambda_{2}\right) \phi^{2}(z)$, it follows that tilting cannot occur until $\Lambda_{2} \geq \Lambda_{0}$, denoted by the dotted line in Fig. 9 .

Although the tilting instability preempts the occurrence of folded brushes, Fig. 10(a) reveals behavior reminiscent of the microphase-segregated brush (MSB) of Birshtein and coworkers ${ }^{33-36}$ just prior to the spinodal of our most flexible chains. In particular, it shows a pronounced concentration for the inner half of the brush relative to that for the outer half. The distributions of individual segments, $\phi(z, s)$, plotted in Fig. 10(b) illustrate that the first half of a chain (i.e., $s \lesssim 0.5$ ) is strongly aligned with the $z$ axis, whereas the second 

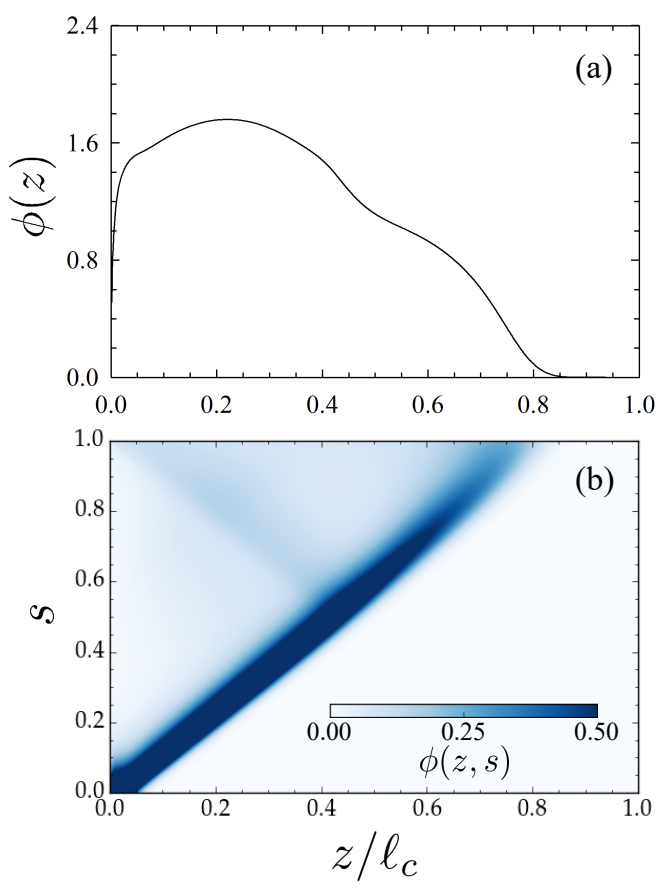

Figure 10: (a) Concentration profile, $\phi(z)$, and (b) $s$-segment distribution, $\phi(z, s)$, for an extended brush of relatively flexible polymers, $\ell_{c}=16 \ell_{p}$, just prior to the spinodal, $\Lambda_{0}=20$ and $\Lambda_{2}=71$

half (i.e., $s \gtrsim 0.5)$ is much less so. It is also evident that the enhanced concentration of the inner brush is due to a small population of folded chains. Evidently, the entropy gained by having two distinct populations is enough to compensate for a few folds, provided the chains are not too stiff.

\section{Discussion}

The tractability of our study has benefitted greatly from the standard semi-dilute approximation, which reduces the number of system parameters to three: $\Lambda_{0}, \Lambda_{2}$, and $\ell_{c} / \ell_{p}$. However, this approximation does prevent us from solving for the equilibrium properties of the tilted phase. Once the energy density, $U(z)$, become sufficiently negative to favor tilting as a means of increasing $\phi(z)$, there is nothing to stop the progression. One needs the higher-order terms in Eq. (8) for the solvent entropy, $S_{s}$, in order to limit the degree of tilting.

This can be illustrated by including the cubic term of $S_{s}$ in $U(z)$, and considering a 
fully-ordered nematic brush (i.e., $\mathcal{S}=1$ and $\mathcal{P}=0$ ) of uniform tilt, $\theta_{\mathbf{n}}$. The cubic term adds

$$
\frac{\Delta U(z)}{n_{p} k_{\mathrm{B}} T}=\frac{\Lambda_{0}^{\prime}}{3} \phi^{3}(z)
$$

to Eq. (20), where $\Lambda_{0}^{\prime}=N \sigma^{2} / 2 b^{2} v_{s} \rho_{0}^{3}$. The assumption of perfect nematic order implies a step profile of concentration $\phi(z)=1 / \cos \left(\theta_{\mathbf{n}}\right)$ and height $\ell_{c} \cos \left(\theta_{\mathbf{n}}\right)$. Thus, the free energy of the brush takes the simple form

$$
\frac{F}{n_{p} k_{\mathrm{B}} T}=-\frac{\delta \Lambda}{2 \cos \left(\theta_{\mathbf{n}}\right)}+\frac{\Lambda_{0}^{\prime}}{3 \cos ^{2}\left(\theta_{\mathbf{n}}\right)}
$$

where $\delta \Lambda \equiv \Lambda_{2}-\Lambda_{0}$. It immediately follows that the extended phase (i.e., $\theta_{\mathbf{n}}=0$ ) remains stable up to the spinodal,

$$
\delta \Lambda_{c}=\frac{4 \Lambda_{0}^{\prime}}{3}
$$

beyond which the equilibrium angle,

$$
\theta_{\mathbf{n}}=\cos ^{-1}\left(\delta \Lambda_{c} / \delta \Lambda\right)
$$

increases continuously. Hence, the spinodal coincides with a continuous tilting transition.

It is evident from Eq. (27) that the inclusion of $\Delta U(z)$ pushes the tilting instability in Fig. 9 towards slightly larger $\Lambda_{2}$. For less ordered brushes (i.e., $\mathcal{S}<1$ ), the weaker LC interactions can be accounted for by making the substitution $\Lambda_{2} \rightarrow \Lambda_{2} \mathcal{S}^{2}$. This shifts the spinodal to larger $\Lambda_{2}$ by a factor of $\mathcal{S}^{-2}$, which is indeed qualitatively consistent with our diagram in Fig. 9. However, we should also account for the fact that tilting increases the nematic order, as demonstrated in Fig. 8(a). This is best done using a conventional Landau expansion, where $F$ is expanded in even powers of $\theta_{\mathbf{n}}$. (Note that odd powers are excluded due to the symmetry between positive and negative tilts.) In this spirit, the angular dependence of the uniaxial order parameter assumes the form $\mathcal{S} \approx \mathcal{S}_{0}+C \theta_{\mathbf{n}}^{2}$, where $C>0$. Expanding Eq. (26) with $\delta \Lambda \equiv \Lambda_{2} \mathcal{S}^{2}-\Lambda_{0}$ is straightforward, albeit a bit involved. For 
small $C$, the spinodal, which is determined by equating the $\theta_{\mathbf{n}}^{2}$ coefficient to zero, simply shifts to smaller $\Lambda_{2}$. However, as $C$ becomes large, the $\theta_{\mathbf{n}}^{4}$ coefficient changes from positive to negative, which causes the tilting transition to switch from second to first order. Thus, for small $\Lambda_{0}$ or large $\ell_{c} / \ell_{p}$, we expect the spinodal to be preempted by a discontinuous tilting transition.

In light of our findings, there are a considerable number of previous studies that ought to be reconsidered, specifically those where strong LC interactions cause the brush to collapse by way of chain folding. In such cases, it is essential to account for the penalty of hairpin folds and to allow the nematic director, $\mathbf{n}(z)$, the freedom to tilt. Some of the phase behavior predicted in the previous studies is likely to be replaced by tilted phases. This includes, for example, the much discussed microphase-segregated brushes (MSB). ${ }^{33-37}$ Although Fig. 10 suggests that the MSB might preempt the tilting instability for sufficiently flexible polymers, this is also the condition for which the tilted phase is expected to preempt the spinodal. Therefore, it will be necessary to actually compare the free energies of the tilted and MSB phases to know for sure which is stable.

Extending the SCFT for worm-like chains to tilted phases will be challenging. First of all, one needs to improve upon the semi-dilute approximation. Including just the cubic term for $S_{s}$ adds one more system parameter, specifically the $\Lambda_{0}^{\prime}$ from Eq. (25). However, this approximation would still breakdown at high concentrations, and so it is probably best to use the full expression for $S_{s}$. This increases the number of system parameters to five: the scaled isotropic interaction parameter, $\nu_{0} N$, the scaled anisotropic interaction parameter, $\nu_{2} N$, the polymer flexibility, $\ell_{c} / \ell_{p}$, the dimensionless grafting density, $\sigma / b \rho_{0}$, and the relative size of the solvent molecules, $v_{s} \rho_{0} / N$. In addition to the large parameter space, the numerics of solving the self-consistent field, $w(z, \mathbf{u})$, for tilted phases will be computationally demanding. When confronted with this problem, Tang et al. ${ }^{50}$ resorted to solving the statistical mechanics of the single worm-like chain in $w(z, \mathbf{u})$ using Monte Carlo simulations. Nevertheless, this challenge can likely be overcome with new improved numerical methods. ${ }^{58}$ We leave this for 
a future study.

The previous study of worm-like chains by Deng et al. ${ }^{43}$ constrained the orientation of $\mathbf{n}$, but in any case their Onsager interaction, $f\left(\mathbf{u}, \mathbf{u}^{\prime}\right)=\epsilon\left|\mathbf{u} \times \mathbf{u}^{\prime}\right|,{ }^{46,51}$ was insufficient to cause the brush to collapse. An expansion of the Onsager interaction in terms of $P_{n}\left(\mathbf{u} \cdot \mathbf{u}^{\prime}\right)$ gives $\nu_{2}=\frac{5}{8} \nu_{0},{ }^{59}$ which according to Fig. 9 puts the system well in the extended brush regime. This can be physically understood by noting that the Onsager interaction accounts for the entropy loss due to the excluded volume of cylindrically-shaped polymers, and therefore always favors low concentrations. One shortcoming of the Onsager interaction is that it overlooks the enthalpic interactions (e.g., van der Waals forces). More seriously, it neglects interactions involving the solvent as well as the solvent entropy, $S_{s}$, both of which contribute to the excluded-volume parameter, $\nu_{0}$. This is inconsequential in bulk phases, because $\phi(z)$ is uniform and thus the value of $\nu_{0}$ has no impact. However, this is not the case for polymer brushes, which is why we used the more general Maier-Saupe interaction energy.

Although the SCFT for worm-like chains tends to be rather computational, it is also very versatile. Once the solvent is treated explicitly, it is straightforward to switch to an LC solvent, and to allow for distinct solvent-solvent, solvent-polymer, and polymer-polymer interactions. The grafting function, $g(z, \mathbf{u})$, can also include any desired dependence on the orientation of the grafted segment, so as to address the tilting transition proposed by Halperin and Williams. ${ }^{17,18}$ Furthermore, the model can be made more realistic. For instance, mainchain liquid crystals, which involve rigid mesogenic segments joined together by relatively flexible linkers, ${ }^{17}$ can be modeled by allowing the bending modulus, $\kappa$, to alternate as a function of $s$ between a finite value for the linkers and infinity for the mesogens. SCFT can readily handle branched architectures, and therefore it can also be extended to side-chain liquid crystals, where mesogen units are grafted to a semi-flexible backbone. ${ }^{19-22,27}$ Provided the brush remains homogeneous in the lateral directions, the computational cost of such extensions will be relatively modest. 


\section{Summary}

We have investigated semi-dilute main-chain LC polymer brushes in good solvent, using SCFT for worm-like chains with Maier-Saupe interactions. The calculation assumes sufficient overlap among the polymers such that the brush has no lateral structure (i.e., no dependence on $x$ and $y$ ), but a low enough polymer concentration such that the solvent can be treated implicitly. Under these assumptions, the grafting density and solvent size can be scaled out of the problem, reducing the number of system parameters to three: the scaled isotropic interaction strength $\Lambda_{0}$, the scaled anisotropic interaction strength $\Lambda_{2}$, and the number of persistence lengths per chain $\ell_{c} / \ell_{p}$. Our study focused on good solvent conditions that favor an extended brush (i.e., $\left.\Lambda_{0}>0\right)$ and LC interactions becoming strong enough to collapse the brush (i.e., $\Lambda_{2} \gtrsim \Lambda_{0}$ ) for polymers ranging from semi-flexible (i.e., $\ell_{c} / \ell_{p}=16$ ) to relatively rigid (i.e., $\ell_{c} / \ell_{p}=1$ ).

The study began by considering azimuthally-symmetric solutions, $w_{0}\left(z, u_{z}\right)$, of the field Eq. (11). Under this constraint, the extended brush collapses by a series of first-order folding transitions, as the LC interactions become strong enough to compensate for an increasing number of folds per chains. However, these transitions overlook the fact that the brush can increase its concentration without the cost of hairpin folds, by instead tilting. Indeed, when the constraint is relaxed, the extended brush becomes unstable with respect to tilting prior to the folding transitions. Within the semi-dilute approximation, the brush concentration increases indefinitely, and thus it becomes necessary treat the solvent entropy, Eq. (8), more accurately. Doing so will produce a second-order tilting transition coinciding with the spinodal, which will likely switch to a first-order transition preempting the spinodal as the polymers become increasingly flexible.

In light of our new findings, it would be prudent to reconsider previous studies that omitted the penalty for hairpin folds and/or assumed a nematic director normal to the grafting surface. Although the SCFT for worm-like chains will become rather computational for tilted phases, it should still be manageable provided the lateral symmetry is retained. The 
reward of using this approach is its remarkable versatility. For example, generalizations to multicomponent brushes, orientational-dependent grafting conditions, and LC solvents are trivial. The model can even be extended, with relatively little additional cost, to more elaborate architectures such as main-chain LC polymers consisting of rigid mesogens connected by semi-flexible segments or side-chain LC polymers consisting of a semiflexible backbone with mesogen side-groups. As such, the SCFT for worm-like chains should be able to handle most systems that experimentalists are likely to study.

\section{Acknowledgments}

We thank Russell Spencer for helping code some of the SCFT. This work was supported by NSERC of Canada, and computer resources were provided by Compute Canada.

\section{References}

(1) Milner, S. T. Polymer Brushes. Science 1991 251, 905-914.

(2) Zhao, B.; Brittain, W. J. Polymer Brushes: Surface-Immobilized Macromolecules. Prog. Polym. Sci. 2000 25, 677-710.

(3) Chen, W.-L.; Cordero, R.; Tran, H.; Ober, C. K. Polymer brushes: Novel Surfaces for Future Materials. Macromolecules 2017, 50, 4089-4113.

(4) Alexander, S. Adsorption of Chain Molecules with a Polar Head - A Scaling Description. J. Phys. France 1977, 38, 983-987.

(5) de Gennes, P. G. Conformations of Polymers Attached to an Interface. Macromolecules 1980, 13, 1069-1075.

(6) Milner, S. T.; Witten, T. A.; Cates, M. E. Theory of Grafted Polymer Brush. Macromolecules 1988, 21, 2610-2619. 
(7) Milner, S. T. Strong-Stretching and Scheutjens-Fleer Descriptions of Grafted Polymer Brushes. J. Chem. Soc. Faraday Trans. 1990, 86, 1349-1353.

(8) Kim, J. U.; Matsen, M. W. Finite-Stretching Corrections to the Milner-Witten-Cates Theory for Polymer Brushes. Eur. Phys. J. E 2007, 23, 135-144.

(9) Pépin, M. P.; Whitmore, M. D. Monte Carlo and Numerical Self-Consistent Field Study of End-Tethered Polymers in Good Solvent. J. Chem. Phys. 1999, 111, 10381-10388.

(10) Kreer, T.; Metzger, S.; Müller, M.; Binder, K.; Baschnagel, J. Static Properties of End-Tethered Polymers in Good Solution: A Comparison between Different Models. J. Chem. Phys. 2004, 120, 4012-4023.

(11) Cosgrove, T.; Heath, T. G.; Phipps, J. S.; Richardson, R. M. Neutron Reflectivity Studies of Polymers Adsorbed on Mica from Solution. Macromolecules 1991, 24, 9498.

(12) Field, J. B.; Toprakcioglu, C.; Ball, R. C.; Stanley, H. B.; Dai, L.; Barford, W.; Penfold, J.; Smith, G.; Hamilton, W. Determination of End-adsorbed Polymer Density Profiles by Neurton Reflectometry. Macromolecules 1992, 25, 434-439.

(13) Kent, M. S.; Lee, L. T.; Farnoux, B.; Rondelez, F.; Characterization of Diblock Copolymer Monolayers at the Liquid-Air Interface by Neutron Reflectivity and Surface Tension Measurements. Macromolecules 1992 25, 6240-6247.

(14) Kent, M. S.; Lee, L. T.; Factor, B. J.; Rondelez, F.; Smith. G. S. Tethered Chains in Good Solvent Conditions: An Experimental Study Involving Langmuir Diblock Copolymer Monolayers. J. Chem. Phys. 1995, 103, 2320-2342.

(15) Baranowski, R.; Whitmore, M. D. Theory of the Structure of Adsorbed Diblock Copolymers: Detailed Comparison with Experiment. J. Chem. Phys. 1995, 103, 2343-2353. 
(16) Pickett, G. T.; Witten, T. A. End-Grafted Polymer Melt with Nematic Interaction. Macromolecules 1992, 25, 4569-4574.

(17) Halperin, A.; Williams, D. R. M. Liquid Crystalline Brushes: an Anchoring Transition. Europhys. Lett. 1993, 21, 575-580.

(18) Halperin, A.; Williams, D. R. M. Liquid Crystalline Polymers in Nematic Solvents: Interfacial Behaviour and Active Anchoring. J. Phys.: Condens. Matter 1994, 6, A297A300.

(19) Peng, B.; Johannsmann, D.; Rühe, J. Polymer Brushes with Liquid Crystalline Side Chains. Macromolecules 1999, 32, 6759-6766.

(20) Peng, B.; Johannsmann, D.; Rühe, J. Homogeneously Aligned Liquid-Crystal Polymer Brushes. Adv. Mater. 2000, 12, 821-824.

(21) Benmouna, F.; Peng, B.; Gapinski, J.; Patkowski, A.; Rühe,; J. Johannsmann, D. Dynamic Light Scattering from Liquid Crystal Polymer Brushes Swollen in a Nematic Solvent. Liquid Crystals 2001, 28, 1353-1360.

(22) Hamelinck, P. J.; Huck, W. T. S. Homotropic Alignment on Surface-Initiated Liquid Crystalline Polymer Brushes. J. Mater. Chem. 2005, 15, 381-385.

(23) Uekusa, T.; Nagano, S.; Seki, T. Unique Molecular Orientation in a Smectic Liquid Crystalline Polymer Film Attained by Surface-Initiated Graft Polymerization. Langmuir 2007, 23, 4642-4645.

(24) Vlasova, E.; Volchek, B.; Tarasenko, I.; Vlasov, G. Spectroscopic Investigation of Polypeptide Plane Brushes. Macromol. Symp. 2011, 305, 116-121.

(25) Haque, H. A.; Hara, M.; Nagano, S.; Seki, T. Photoinduced In-Plane Motions of Azobenzene Mesogens Affected by the Flexibility of Underlying Amorphous Chains. Macromolecules 2013, 46, 8275-8283. 
(26) Sato, O; Iwata, N.; Kasaib, T.; Tsujiic, Y.; Kangb, S.; Watanabe, J.; Tokita, M. Nematic Liquid Crystal Anchoring Strengths of High Density Polymer Brush Surfaces. Liquid Crystals 2015, 42, 181-181.

(27) Stetsyshyn, Y.; Raczkowska, J.; Budkowski, A.; Awsiuk, K.; Kostruba, A.; Nastyshyn, S; Harhay, K.; Lychkovskyy, E.; Ohar, H.; Nastishin, Y. Cholesterol-Based Grafted Polymer Brushes as Alignment Coating with Temperature-Tuned Anchoring for Nematic Liquid Crystals. Langmuir 2016, 32, 11029-11038.

(28) Tran, H.; Zhang, Y.; Ober. C. K. Synthesis, Processing, and Characterization of Helical Polypeptide Rod-Coil Mixed Brushes. ACS Macro. Lett. 2018, 7, 1186-1191.

(29) Li, X.; Yanagimachi, T.; Bishop, C.; Smith, C.; Dolejsi, M.; Xie, H.; Kurihara, K.; Nealey, P. F. Engineering the Anchoring Behavior of Nematic Liquid Crystals on a Solid Surface by Varying the Density of Liquid Crystalline Polymer Brushes. Soft Matter 2018, 14, 7569-7577.

(30) Birshtein, T. M.; Mercurieva, A. A.; Pryamitsyn, V. A.; Polotzkiy, A. Liquid-Crystalline Ordering in Polymer Brushes. Macromol. Theory Simul., 1996, 5, 215-223.

(31) Maier, W.; Saupe, A. Eine Einfache Molekulare Theorie des Nematischen Kristallinflussigen Zustandes. Z. Naturforsch., A 1958, 13, 564-566.

(32) Kuznetsov, D. V.; Chen, Z. Y. Semiflexible Polymer Brushes: A Scaling Theory. J. Chem. Phys. 1998, 109, 7017-7027.

(33) Amoskov, V. M.; Birshtein, T. M.; Pryamitsyn, V. A. Theory of Polymer Brushes of Liquid-Crystalline Polymers. Macromolecules 1996, 29, 7240-7250.

(34) Birshtein, T. M.; Amoskov, V. M.; Mercurieva, A. A.; Pryamitsyn, V. A. Phase Transitions in Polymer Brushes. Macromol. Symp. 1997, 113, 151-161. 
(35) Darinskii, A. A.; Tupitsyna, A. I.; Birshtein, T. M.; Saf'yannikova, M. G.; Amoskov, V. M.; Emri, I. Microphase Separation in Brushes Capable of Liquid Crystal Ordering. Polym. Sci. A 2003, 45, 665-675.

(36) Mercurieva, A. A.; Birshtein, T. M.; Amoskov, V. M. Theory of Liquid-Crystalline Ordering in Polymer Brushes. Macromol. Symp. 2007, 252, 90-100.

(37) Tupitsyna, A. I.; Darinskii, A. A.; Emri, I.; Allen, M. P. Computer Simulation of Anisotropic Polymer Brushes. Soft Matter 2008, 4, 108-121.

(38) Williams, D. R. M.; Warner, M. Statics and Dynamics of Hairpins in Worm-Like Main Chain Nematic Polymer Liquid Crystals. J. Phys. France 1990, 51, 317-339.

(39) Lange, H.; Schmid, F. Surface Anchoring on Liquid Crystalline Polymer Brushes. Comp. Phys. Commun. 2002, 147, 276-281.

(40) Lange, H.; Schmid, F. Surface Anchoring on Layers of Grafted Liquid-Crystalline Chain Molecules: A Computer Simulation. J. Chem. Phys. 2002, 117, 362-368.

(41) Lange, H.; Schmid, F. An Anchoring Transition at Surfaces with Grafted LiquidCrystalline Chain Molecules. Eur. Phys. J. E 2002, 7, 175-182.

(42) Downton, M. T.; Allen, M. P. Computer Simulation of Liquid-Crystal Surface Modification. Europhys. Lett. 2004, 65, 48-54.

(43) Deng, M.; Jiang, Y.; Liang, H.; Chen, J. Z. Y. Wormlike Polymer Brush: A SelfConsistent Field Treatment. Macromolecules 2010, 43, 3455-3464.

(44) Kratky, O.; Porod, G. Röntgenuntersuchung Gelöster Fadenmoleküle. Recl. Trav. Chem. Pays-Bas 1949, 68, 1106-1123.

(45) Saito, N.; Takahashi, K.; Yunoki, Y. Statistical Mechanical Theory of Stiff Chains. J. Phys. Soc. Jpn. 1967, 22, 219-226. 
(46) Onsager, L. The Effects of Shape on the Interaction of Colloidal Particles. Ann. N.Y. Acad. Sci. 1949, 51, 627-659.

(47) Polotsky, A. A.; Birshtein, T. M. Normal and Lateral Deformation of Lyotropically Ordered Polymer Brush. Macromol. Theory Simul. 2006, 15, 654-667.

(48) Milchev, A.; Binder, K. Bending or Buckling: Compression-Induced Phase Transition in a Semi-Flexible Polymer Brush. Europhys. Lett. 2013, 102, 58003.

(49) Milchev, A.; Binder, K. Unconventional Ordering Behavior of Semi-Flexible Polymers in Dense Brushes Under Compression. Soft Matter 2014, 10, 3783-3797.

(50) Tang, J.; Zhang, X.; Yan, D. Compression Induced Phase Transition of Nematic Brush: A Mean-Field Theory Study. J. Chem. Phys. 2015, 143, 204903.

(51) Fredrickson, G. H. The Equilibrium Theory of Inhomogeneous Polymers; Oxford University Press: New York, 2006.

(52) Morse, D. C.; Fredrickson, G. H. Semiflexible Polymers near Interfaces. Phys. Rev. Lett. 1994, 73, 3235-3238.

(53) de Gennes, P. G.; Prost, J. The Physics of Liquid Crystals, 2nd Ed.; Clarendon, Oxford, 1993.

(54) Daoulas, K. C.; Theodorou, D. N.; Harmandaris, V. A.; Karayiannis, N. C.; Mavrantzas, V. G. Self-Consistent-Field Study of Compressible Semiflexible Melts Adsorbed on a Solid Substrate and Comparison with Atomistic Simulations. Macromolecules 2005, 38, 7134-7149.

(55) Anderson, D. G. Iterative Procedures for Nonlinear Integral Equations. J. Assoc. Comput. Mach. 1965, 12, 547-560. 
(56) Matsen, M. W. Fast and Accurate SCFT Calculations for Periodic Block-Copolymer Morphologies using the Spectral Method with Anderson Mixing. Eur. Phys. J. E 2009, 30, 361-369.

(57) Stasiak, P.; Matsen, M. W. Efficiency of Pseudo-Spectral Algorithms with Anderson Mixing for the SCFT of Periodic Block-Copolymer Phases. Eur. Phys. J. E 2011, 34, 110.

(58) Hannon, A.F.; Kline, J.R.; DeLongchamp, D. Advancing the Computational Methodology of Rigid Rod and Semiflexible Polymer Systems: A New Solution to the Wormlike Chain Model with Rod-Coil Copolymer Calculations. J. Polym. Sci., Part B, 2019, $57,29-39$.

(59) Cui, S.-M.; Akcakir, O.; Chen, Z. Y. Isotropic-Nematic Interface of Liquid-Crystalline Polymers. Phys. Rev. E 1995, 51, 4548-4557. 


\section{for Table of Contents use only}

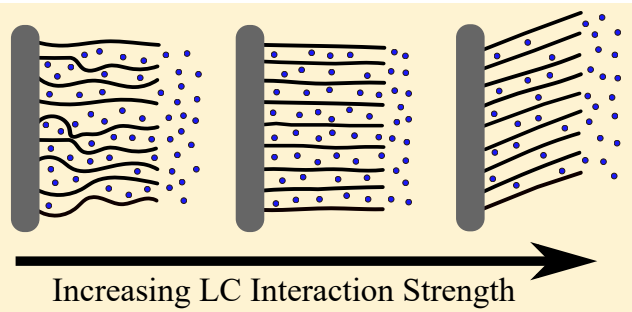

Title: Spontaneous Tilting Transition in Liquid-Crystalline Polymer Brushes

Authors: S. Blaber, N.M. Abukhdeir, and M.W. Matsen 